\title{
A NUMBER OF CONFLICTS AT ROUTE INTERSECTIONS - RECTANGULAR MODEL
}

\begin{abstract}
A conflict is an infringement of minimum separation between at least two aircraft. Air traffic controllers build a protected area in front of an aircraft; its shapes and dimensions depend on the speed of an aircraft and on the minimum separation. The protected zone in this mathematical model is the horizontal rectangular zone. If an aircraft is inside this protected zone, a conflict occurs. The model is based on these assumptions: aircraft fly in level straight line routes; only an infringement of the lateral separation is considered; deviations are excluded; aircraft at the same flight level fly at the same average speed; aircraft fly towards an intersection and change direction after the intersection. Hence, conflicts mainly occur owing to the loss of minimum separation between aircraft flying at the same flight level. The calculation of average number of potential conflicts is designed for a long time interval; hence, aircraft velocity deviations are negligible. The mathematical model in this paper is intended to compare different alternative intersection configurations of air traffic service routes. The comparison is based on the following results: an average number of potential conflicts per hour at route intersections, index of conflict intensity, and intersection capacity.

Keywords: Intersection configuration, aircraft conflicts, horizontal separation, protected zone.
\end{abstract}

\section{Introduction}

Air transport significantly contributes to the world economy development. Therefore, it is very important to sustain its further resilience, ensure effective, ecological activities and mainly ensure its safety.

In 2015, more than 3.5 billion passengers used scheduled air transport. When compared with 2014, the growth was $6.4 \%$. The number of flights worldwide reached 34 million.

Based on the air traffic development the ICAO identified the Performance Based Navigation (PBN) as the main global priority. The ICAO concentrated as well on the PBN implementation at international airports during Continuous Descent Operations and Continuous Climb Operations. The SESAR AMAN and DMAN concepts, Free Route Airspace and growing air traffic require delineation of new routes [1].

All the above facts lead to the need of research of conflicts at route intersections in order to prevent them. The mathematical model presented in the paper "A number of conflicts at route intersections - a rectangular model" enables comparing different alternatives of intersection configuration of air traffic services routes. The comparison is based on the results: average number of potential conflicts per hour at route intersections, index of conflict intensity, and intersection capacity. The results are intended to help choose the safest intersection configuration of routes.

The consequences of a particular conflicts depend on the design of item and the equipment in which it is install. Although the impairment in which the equipment is operated is sometimes an additional factor conflict consequences are primary inherent characteristic [2].

A conflict is an event in which two or more aircraft experience a loss of minimum separation. A conflict occurs when the distance between aircraft in flight violates a prescribed minimum, usually considered as 5 nautical miles $(9 \mathrm{~km})$ of horizontal and/or 1000 feet of vertical separation in radar environment [3]. These distances define an aircraft's protected zone, a volume of airspace surrounding the aircraft which should not be infringed upon by any other aircraft.

Aircraft operating on cruising levels fly horizontal trajectories and are separated vertically, it follows that conflicts mainly occur owing to a loss of minimum separation between aircraft flying at the same flight level.

\section{Model}

A conflict situation in a radar environment occurs when the radar separation between aircraft is less than the prescribed

\footnotetext{
* Karel Havel, Vojtech Balint, Andrej Novak

Faculty of Operation and Economics of Transport and Communications, University of Zilina, Slovakia

E-mail: andrej.novak@fpedas.uniza.sk
} 
minimum. However, air traffic controllers (ATCOs) build in front of an aircraft a protected zone, its dimensions depend on the speed of an aircraft and on the required minimum separation [4]. The protected zone, in this mathematical model, will be the rectangular zone; its dimensions are $l$ which is a length of the protected zone and $w$ which is a width of the protected zone. If an aircraft intrudes this protected zone a conflict occurs. Values $l$ and $\mathrm{w}$ may be increased due to possible aircraft deviations from routes [5]. The mathematical model is intended to compare different alternatives of an intersection configuration. The assumptions and discussion above can be summed up as follows [6]:

1. Aircraft fly in level flight and at flight levels

2. Only infringement of the lateral separation is considered

3. Aircraft fly on the route which is a straight line, deviations are excluded

4. Aircraft at the same flight level fly at the same average speed

5. Longitudinal separation is always assured by ATC

6. Aircraft fly towards an intersection and change direction after the intersection.

\subsection{Legend}

We denote:

$l$ - length of the protected zone,

$w$ - width of the protected zone,

$\bar{a}$ - aircraft on the route, $A P B$,

$\bar{b}$ - aircraft on the route, $C P D$,

$\delta$ - angle of arrival at the intersection $P$,

$\beta$ - oriented angle, means the change of direction of flight of aircraft $\bar{b}$ in $P$,

$\alpha$ - oriented angle, means the change of direction of flight of aircraft $\bar{a}$ in $P$,

$V$ - speed of both aircraft (constant and the same).

The angles are positive if they are anticlockwise.

\subsection{Deriving the Model}

We assume that aircraft $\bar{b}$ is in point $P$. The suggested model will establish the position of aircraft $\bar{a}$ in order to

- avoid conflict of $\bar{a}, \bar{b}$ in the particular moment

- avoid conflict of $\bar{a}, \bar{b}$ in the past (assuming backward time shift),

- avoid conflict of $\bar{a}, \bar{b}$ in the future (assuming forward time shift).

The model attempts to determine a critical zone in front of $P$ (denoted $L P$ ) and behind $P$ (denoted $P M$ ). Assuming aircraft $\bar{a}$ avoids the critical zone and aircraft $\bar{b}$ is in $P$, there is, was or will be no risk of conflict. However, if aircraft $\bar{a}$ is in segment $L P$ or $P M$, it will inevitably result in a conflict.
The whole situation is illustrated in Fig. 1. First, we will define critical zone in front of $P$.

If aircraft $\bar{b}$ is in $R$, the protected zone front part of aircraft $\bar{a}$ can be on the left of $N$, which is a perpendicular projection of $R$ on $A P$. If we consider backward time shift of both aircraft so that $\bar{b}$ is in $P$, the protected zone front part of aircraft $\bar{a}$ is in a such point $U$ left of $N$ that $|U N|=|R P|$ . Therefore, aircraft $\bar{a}$ must be in $L$ left of $U$ and $|L U|=l$.

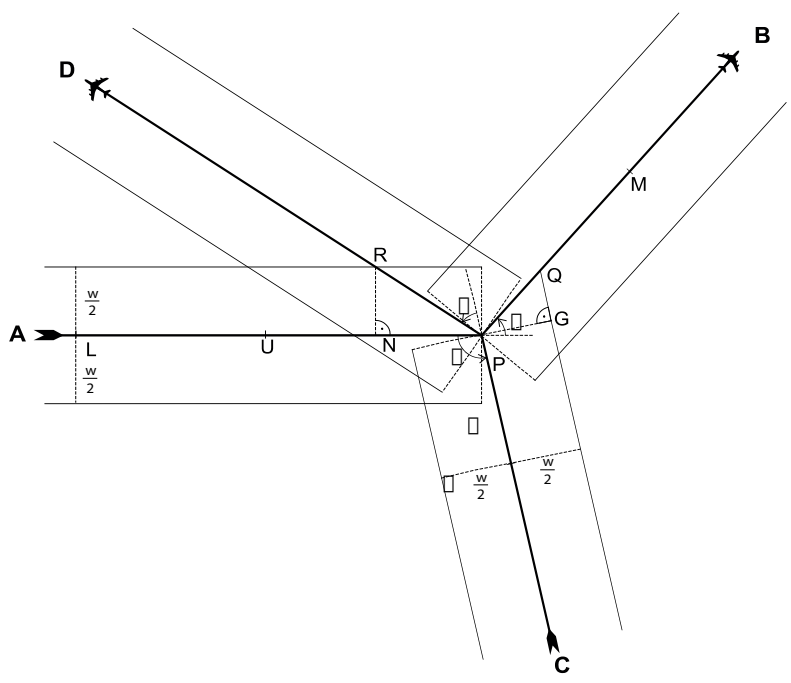

Fig. 1 Intersection

From the triangle PNR follows that angle $\angle N P R=\pi-\delta-\beta$, and therefore

$|R P|=\frac{w}{2} \cdot \frac{1}{\sin (\pi-\delta-\beta)}=\frac{w}{2} \cdot \frac{1}{\sin (\delta+\beta)}$ and

$|N P|=\frac{w}{2} \cdot \operatorname{cotg}(\pi-\delta-\beta)=-\frac{w}{2} \cdot \operatorname{cotg}(\delta+\beta)$.

Hence

$|L P|=|L U|+|U N|+|N P|=l+\frac{w}{2} \cdot \frac{1}{\sin (\delta+\beta)}-$

$-\frac{w}{2} \cdot \operatorname{cotg}(\delta+\beta)=l+\frac{w}{2} \cdot \frac{1-\cos (\delta+\beta)}{\sin (\delta+\beta)}=$

$=l+\frac{w}{2} \cdot \operatorname{tg} \frac{\delta+\beta}{2}$

Let us find the critical zone behind point $P$. If the protected zone front part of aircraft $\bar{b}$ is in $Q$, i.e. the distance between $\bar{b}$ and $P$ is $l-|Q G|$, aircraft $\bar{a}$ must be right of $Q$. Assuming the forward time shift, aircraft $\bar{b}$ will be in $P$, aircraft $\bar{a}$ must be in such $M$ that $|Q M|=l-|Q G|$.

In the right-angled triangle $Q G P$ angle

$\angle Q G P=\frac{\pi}{2}+\alpha-\delta$, therefore 


$$
\begin{aligned}
& |Q G|=\frac{w}{2} \cdot \operatorname{tg}\left(\frac{\pi}{2}+\alpha-\delta\right)=-\frac{w}{2} \cdot \operatorname{cotg}(\alpha-\delta) \\
& |P Q|=\frac{w}{2} \cdot \frac{1}{\cos \left(\frac{\pi}{2}+\alpha-\delta\right)}=-\frac{w}{2} \cdot \frac{1}{\sin (\alpha-\delta)}
\end{aligned}
$$

Hence

$$
\begin{aligned}
& |P M|=|P Q|+|Q M|=|P Q|+l-|Q G|= \\
& =-\frac{w}{2} \cdot \frac{1}{\sin (\alpha-\delta)}+l+\frac{w}{2} \cdot \operatorname{cotg}(\alpha-\delta)= \\
& l+\frac{w}{2} \cdot \frac{1-\cos (\alpha-\delta)}{\sin (\alpha-\delta)}=l-\frac{w}{2} \cdot \operatorname{tg} \frac{\alpha-\delta}{2}= \\
& =l+\frac{w}{2} \operatorname{tg} \frac{\delta-\alpha}{2} .
\end{aligned}
$$

Thus the length of the critical zone is

$$
\begin{aligned}
& |L M|=|L P|+|P M|=l+\frac{w}{2} \cdot \operatorname{tg} \frac{\delta+\beta}{2}+l+ \\
& +\frac{w}{2} \cdot \operatorname{tg} \frac{\delta-\alpha}{2}=2 l+\frac{w}{2} \cdot\left(\operatorname{tg} \frac{\delta+\beta}{2}+\operatorname{tg} \frac{\delta-\alpha}{2}\right) .
\end{aligned}
$$

\subsection{Average number of potential conflicts per hour}

If the average speed of both aircraft is $V$, the time of flight on $L M$ is $L M / V$.

Let us denote:

$f_{1}$ - average traffic flow on $A P B$,

$f_{2}$ - average traffic flow on $C P D$.

Hence, the average occupancy time $T$ of aircraft from flow $f_{1}$ on segment $L M$ is

$$
T=\frac{f_{1} L M}{V}=\frac{f_{1}}{V}\left(2 l+\frac{w}{2}\left(\operatorname{tg} \frac{\delta-\alpha}{2}+\operatorname{tg} \frac{\delta+\beta}{2}\right)\right) .
$$

During the time $T$ we can expect $T f_{2}$ aircraft from the flow $f_{2}$. This value $T f_{2}$ is obviously an average number $E$ of potential conflicts per hour
$E=T f_{2}=\frac{f_{1} f_{2}}{V}\left(2 l+\frac{w}{2}\left(\operatorname{tg} \frac{\delta-\alpha}{2}+\operatorname{tg} \frac{\delta+\beta}{2}\right)\right)$.

\section{INDEX OF CONFLICT INTENSITY}

It describes the intersection $I$ without the influence of traffic flows

$I=\frac{E}{f_{1} f_{2}}=\frac{1}{V}\left(2 l+\frac{w}{2}\left(\operatorname{tg} \frac{\delta-\alpha}{2}+\operatorname{tg} \frac{\delta+\beta}{2}\right)\right)$.

\section{CAPACITY OF INTERSECTION}

The equation above can be modified to the following expression

$$
f_{1} f_{2}=\frac{E V}{2 l+\frac{w}{2}\left(\operatorname{tg} \frac{\delta-\alpha}{2}+\operatorname{tg} \frac{\delta+\beta}{2}\right)} .
$$

\section{Conclusion}

The mathematical model is designed to compare different alternatives of intersection configuration. The evaluation and comparison is based on an average number of potential conflicts per hour, index of conflict intensity, capacity of intersection. Calculation of average number of potential conflicts is designed for a long time interval; hence aircraft velocity deviations are negligible. Average number of potential conflicts per hour depends mainly on average traffic flows on routes. Decisive for index of conflict intensity are rectangular zone dimensions. The number $f_{1} f_{2}$ is a capacity of intersection for a given number of potential conflicts per hour and it mainly depends on an intersection configuration.

\section{Acknowledgement}

This paper is published as one of the scientific outputs of the project: „Centre of Excellence for Air Transport ITMS 26220120065“.

\section{References}

[1] ICAO - Air Navigation Report, Capacity \& Efficiency, 2016.

[2] BUGAJ, M., NOVAK, A., BENO, L.: Application of RCM Principles in the Air Operations. Communications - Scientific Letters of the University of Zilina, No. 2, 2005, 20-62, ISSN 1335-4205.

[3] ICAO Doc 4444 - Air Traffic Management, $15^{\text {th }}$ edition - Code 7700.

[4] HAVEL, K., et al.: Air Traffic Control, Praha : Nadas, 1990, ISBN 80-7030-056-6.

[5] BADANIK, B., BARTOS, M.: Innovative Approach in Provision of Air Navigation Services in Europe, Zilinska univerzita : Zilina, 2016, 24-27, ISBN 978-80-554-1252-8.

[6] HAVEL, K., HUSARCIK, J.: A Theory of the Tactical Conflict Prediction of a Pair of Aircraft, J. of Navigation, vol. 42, No. 3, 417-429, 1989, ISSN 0373-4633. 